

\title{
Fault detection and identification via bounded-error parameter estimation using distribution theory
}

\author{
Nathalie Verdière, Carine Jauberthie
}

\section{To cite this version:}

Nathalie Verdière, Carine Jauberthie. Fault detection and identification via bounded-error parameter estimation using distribution theory. International Conference on Control, Decision and Information Technologies (CoDIT 2019), Apr 2019, Paris, France. hal-02151533

\section{HAL Id: hal-02151533 \\ https://hal.science/hal-02151533}

Submitted on 8 Jun 2019

HAL is a multi-disciplinary open access archive for the deposit and dissemination of scientific research documents, whether they are published or not. The documents may come from teaching and research institutions in France or abroad, or from public or private research centers.
L'archive ouverte pluridisciplinaire HAL, est destinée au dépôt et à la diffusion de documents scientifiques de niveau recherche, publiés ou non, émanant des établissements d'enseignement et de recherche français ou étrangers, des laboratoires publics ou privés. 


\title{
Fault detection and identification via bounded-error parameter estimation using distribution theory
}

\author{
Nathalie Verdière ${ }^{1}$ and Carine Jauberthie ${ }^{2}$
}

\begin{abstract}
In this paper, an improvement of the boundederror fault detection and identification method based on inputoutput polynomials of ([2]) is proposed. It is based on integrodifferential polynomials used to estimate the fault values. The standard input-output polynomials are obtained from differential algebra elimination theory and can be used both for diagnosability analysis and fault estimation. Unfortunately, they may involve derivatives of high order whose estimation is a hard problem when system outputs are uncertain. Distribution theory allows us to transform them into integrodifferential polynomials that involve lower order derivatives of the model outputs. In this paper, this method, extended to the set-membership (SM) framework, is used with the focus of achieving fault detection and identification. The original method and the new method are applied to a coupled water-tanks model and compared. It is shown that the new method significantly improves the fault detection and identification results.
\end{abstract}

\section{INTRODUCTION}

Fault detection and identification is at the core of any monitoring system and guarantees availability and safety of the monitored system. Among the methods that allow to achieve these tasks and provide analytical redundancy, the most obvious approach is to estimate the faults considered as parameters of the model. A common case is when faults can be considered as parameter deviations. Parameters can indeed be considered as the representative features of the system's components health status. In this case, given the a priori knowledge of the parameter nominal values, one can then compare them with the estimated values and evaluate the discrepancy. The advantage of this approach is that one achieves at once the two tasks, fault detection and fault identification, since the method returns the values of the fault parameters ([2], [10]). In this paper, we focus on a numerical method proposed in [8]. It is based on Analytical Redundancy Relations (ARRs) obtained from differential algebra tools ([1]) and the use of the distribution theory so that new relations in the form of integro-differential polynomials are obtained. Their main advantage is that the orders of derivatives are lower than those involved in the initial ARRs. In [8], it was proven that better results are obtained for the estimation of parameters.

From classical and integro-differential polynomials, a numerical procedure is implemented for detecting and esti-

\footnotetext{
*This work was not supported by any organization

${ }^{1}$ Verdière $\mathrm{N}$. is with University of Normandie, UNIHAVRE, LMAH, FR-CNRS-3335, ISCN, 76600 Le Havre, France verdiern@univ-lehavre.fr

${ }^{2}$ Jauberthie C. is with LAAS-CNRS, Université de Toulouse, CNRS, UPS, 7 avenue du Colonel Roche, 31400 Toulouse, France cjaubertelaas.fr
}

mating faults in nonlinear models. We adopt a boundederror estimation framework in order to take into account the uncertainties attached to the measures and to guarantee the fault estimation. Indeed, this insures that the set of all values are consistent with the bounds specified for the measures.

In this paper, we take as a starting point the fault detection and identification of multiple faults in two coupled water tanks one above the other and modeled by:

$$
\left\{\begin{array}{l}
\dot{x}_{1}(t, p)=\left(a_{1}+f_{1}\right) u(t)-\left(a_{2}+f_{2}\right) \sqrt{x_{1}(t, p)}, \\
\dot{x}_{2}(t, p)=\left(a_{3}+f_{3}\right) \sqrt{x_{1}(t, p)}-\left(a_{4}+f_{4}\right) \sqrt{x_{2}(t, p)}, \\
y_{1}(t, p)=\sqrt{x_{1}(t, p)}, \\
y_{2}(t, p)=\sqrt{x_{2}(t, p)},
\end{array}\right.
$$

where $p=\left(a_{i}\right)_{i=1, \ldots, 4}, a_{i} \neq 0$, is the model parameter vector, $f=\left(f_{1}, f_{2}, f_{3}, f_{4}\right)$ the unknown additive fault vector such that $0<f_{i}<1$. $x=\left(x_{1}, x_{2}\right)^{T}$ represents the state vector and corresponds to the level in each tank, and $u \not \equiv 0$ is the input vector. The water level in the tanks can vary between 0 and 10 .

The paper is organized as follows. In section II, the fault estimation procedures based on the classical input-output polynomials and then on the integro-differential polynomials are described. Fault detection and identification is then achieved on the water-tank model in section III. Finally section IV concludes the paper.

\section{FAULT DETECTION AND IDENTIFICATION PROCEDURES}

The fault detection and identification procedures proposed in this paper make use of the ARRs found. From them, two estimation methods are proposed in this section. The first method uses directly the ARRs and, from the output systems, estimates its coefficients and consequently the faults. However, ARRs obtained by elimination methods can contain derivatives of high order which are difficult to estimate in a bounded-error context even if some progresses have been done [5]. The second method is based on a pre-treatment of the ARRs consisting in using a distribution approach. More precisely, the polynomials are multiplied by test functions which are smooth functions and the new polynomials are then integrated. This operation permits to report part of the output derivatives on the known test functions whose derivatives can be explicitly calculated. The coefficients of the last polynomials are then estimated. Assuming that the faults act by varying the parameter values and since it is assumed that the nominal parameter values are known within 
specified error bounds, its intersection with the estimated value gives the state of the system: if the intersection is empty, the system is considered faulty and an anomaly is detected.

\section{A. Preliminaries}

We denote $y_{k}=y\left(t_{k}\right)$ the measures done at the discrete times $\left(t_{k}\right)_{1 \leq k \leq M}$, and the associated intputs $u_{k}=u\left(t_{k}\right)$. From these measures, the input-output method consists in estimating the vector fault composed of the so-called parameter blocks $\left(\theta_{j}(f, p)\right)_{j=1, \ldots, q}$ using the polynomial $R(y, u, f, p)$ given by (2).

The measurement noise and uncertainties on parameters are taken into account by assuming that the output $y$ is disturbed by a bounded additive noise $\eta(t) \in[\eta(t)]$, the parameter fault vector $f$ (resp. $p$ ) belongs to $F$ (resp. $P$ ) where $F$ (resp. $P$ ) is an interval vector. Consider $\Theta_{j}(F, P)$ obtained from $\theta_{j}(f, p)$ by substituting $f$ and $p$ by the sets $F$ and $P$ respectively. $\Theta_{j}(F, P)$ is a connected set for all connected sets $F$ and $P$ since it only involves sum, difference and product of connected sets.

For any matrix $A$, we denote $(A)_{k}$ (resp. $[A]_{k}$ ) the $k$ th line of the matrix $A$ (resp. the $k$ th line of the interval matrix $[A]$ ). Afterwards, we suppose that the family of functions $\psi_{i}$ 's, for $i=1, \ldots, M$ are infinitely differentiable functions equal to zero outside the compact interval $\left[t_{i}-\epsilon, t_{i}+\epsilon\right], \epsilon>0$.

We assume too that the component functions of $u, y$, and $x$ verify the following assumption:

(H): they are $C^{\infty}$ in $] 0, T[$ and are equal to zero on $]-\infty, 0[$, ]$T,+\infty[$. They coincide in $] 0, T[$ with functions which are $C^{\infty}$ in $[0, T[$.

In that follows, an enclosure of $\int_{a}^{b} f(s) d s$ in the setmembership framework will be denoted by $I_{[a, b]}(f)$ afterwards.

\section{B. Analytical Redundancy Relations (ARRs)}

ARRs take the form of differential polynomials linking system inputs, outputs and their derivatives that can be obtained using elimination theory. They had already been used to estimate parameters (or faults) when no initial guess of their values were known [6] and for doing fault detection [7]. According to [9], ARRs can take the following form:

$$
R(y, u, f, p)=m_{0}(y, u, p)+\sum_{j=1}^{q} \theta_{j}(f, p) m_{j}(y, u)=0
$$

where $\left(\theta_{j}(f, p)\right)_{1 \leq j \leq q_{i}}$ are rational in $p$ and $f, \theta_{a} \neq \theta_{b}$ $(a \neq b),\left(m_{j}(y, u)\right)_{1 \leq j \leq q}$ are differential polynomials with respect to $y$ and $u$ and $m_{0} \neq 0$.

Example 1: Let come back to the example of the watertank. In order to use the Rosenfeld-Groebner algorithm implemented in Maple 16, auxiliary variables $z_{1}(t, p)=$ $\sqrt{x_{1}(t, p)}$ and $z_{2}(t, p)=\sqrt{x_{2}(t, p)}$ are introduced and the model, including the representation of the four faults, is rewritten as:

$$
\left\{\begin{array}{l}
\dot{x}_{1}(t, p)=\left(a_{1}+f_{1}\right) u(t)-\left(a_{2}+f_{2}\right) z_{1}(t, p) \\
\dot{x}_{2}(t, p)=\left(a_{3}+f_{3}\right) z_{1}(t)-\left(a_{4}+f_{4}\right) z_{2}(t, p) \\
z_{1}(t, p)^{2}=x_{1}(t, p), z_{2}(t, p)^{2}=x_{2}(t, p) \\
y_{1}(t, p)=z_{1}(t, p), y_{2}(t, p)=z_{2}(t, p)
\end{array}\right.
$$

According to the Rosenfeld-Groebner algorithm, we obtain two input-output relations:

$$
\begin{aligned}
& R_{1}(y, u, p)=-a_{1} u+a_{2} y_{1}+2 y_{1} \dot{y}_{1}-f_{1} u+f_{2} y_{1}, \\
& R_{2}(y, u, p)=a_{4} y_{2}-a_{3} y_{1}+2 y_{2} \dot{y}_{2}-f_{3} y_{1}+f_{4} y_{2} .
\end{aligned}
$$

\section{Estimation methods}

Let us recall briefly the two methods (see [8] for more details). The first method consists in using the property that the ARRs (2) are linear with respect to the parameter blocks $\theta_{1}(f, p), \ldots, \theta_{q}(f, p)$ and consequently a linear system can be formed.

The second method is based on the use of the distribution theory. The ARRs (2) are multiplying by the test functions $\psi_{i}$ and the relations are then integrated so that integrodifferential polynomials are obtained. These new relations are linear too with respect to the parameter blocks and a new system is also deduced.

In the two cases, a system of the form $\tilde{A} \theta=\tilde{b}$ is obtained. Considering a bounded error disturbed output, this system can be interpreted in the set-membership framework and used to estimate the unknown interval vector $\left(\Theta_{j}(F, P)\right)_{1 \leq j \leq q}$. It consists in solving the following system

$$
[\tilde{A}][\theta]=[\tilde{b}]
$$

Solving system (4) can be cast using the SIVIA algorithm (Set Inversion Via Interval Analysis) [3] for which it is necessary to give initial intervals. The problem that is solved here is to find $[x]$ such that $0 \in[A][x]-[b]$. We will use the forward-backward propagation to contract the initial parameter box.

Example 2: Method based on input-output polynomials: we denote $y_{1, p}^{(1)}\left(t_{k}\right)$ (resp. $y_{2, p}^{(1)}\left(t_{k}\right)$ ) the estimate of $\dot{y}_{1}\left(t_{k}\right)$ (resp. $\dot{y}_{2}\left(t_{k}\right)$ ). From $R_{1}$ defined at Example 1, the first system which has to be solved is $\left[A_{1}\right][\theta]=\left[b_{1}\right]$ where $\left[A_{1}\right]_{k}=\left(\left[-u\left(t_{k}\right)\right],\left[y_{1}\left(t_{k}\right)\right]\right)$ and $\left[b_{1}\right]_{k}=\left[a_{1} u\left(t_{k}\right)-\right.$ $\left.a_{2} y_{1}\left(t_{k}\right)-2 y_{1}\left(t_{k}\right) y_{1, p}^{(1)}\left(t_{k}\right)\right]$.

The second system is composed of the matrix interval $\left[A_{2}\right]$ and the vector interval $\left[b_{2}\right]$ such that $\left[A_{2}\right]_{i}=\left(\left[-y_{1}\left(t_{i}\right)\right],\left[y_{2}\left(t_{i}\right)\right]\right)$ and $\left[b_{2}\right]_{i}=$ $\left[-a_{4} y_{2}\left(t_{k}\right)+a_{3} y_{1}\left(t_{k}\right)-2 y_{2}\left(t_{i}\right) y_{2, p}\left(t_{i}\right)\right]$.

Method based on integro-differential polynomials: Similarly, the two systems to be solved are $\left[A_{1}^{\psi}\right][\theta]=\left[b_{1}^{\psi}\right]$ and $\left[A_{2}^{\psi}\right][\theta]=\left[b_{2}^{\psi}\right]$ such that

$$
\begin{aligned}
& {\left[A_{1}^{\psi}\right]_{i}=\left(\left[-I_{\left[t_{i}-\epsilon, t_{i}+\epsilon\right]}\left(u \psi_{i}\right)\right],\left[I_{\left[t_{i}-\epsilon, t_{i}+\epsilon\right]}\left(y_{1} \psi_{i}\right)\right]\right),} \\
& {\left[b_{1}^{\psi}\right]_{i}=\left[I_{\left[t_{i}-\epsilon, t_{i}+\epsilon\right]}\left(a_{1} u-a_{2} y_{1}+y_{1}^{2} \dot{\psi}_{i}\right)\right] .}
\end{aligned}
$$

and

$$
\begin{aligned}
& {\left[A_{2}^{\psi}\right]_{i}=\left(\left[-I_{\left[t_{i}-\epsilon, t_{i}+\epsilon\right]}\left(y_{1} \psi_{i}\right)\right],\left[I_{\left[t_{i}-\epsilon, t_{i}+\epsilon\right]}\left(y_{2} \psi_{i}\right)\right]\right)} \\
& {\left[b_{2}^{\psi}\right]_{i}=\left[I_{\left[t_{i}-\epsilon, t_{i}+\epsilon\right]}\left(-a_{4} y_{2}+a_{3} y_{1}+y_{2}^{2} \dot{\psi}_{i}\right)\right] .}
\end{aligned}
$$




\section{APPLICATION}

The test functions $\psi_{i}, i=1, \ldots, M$ are bell-shaped functions centered at $t_{i}$. They are constructed from the test func-

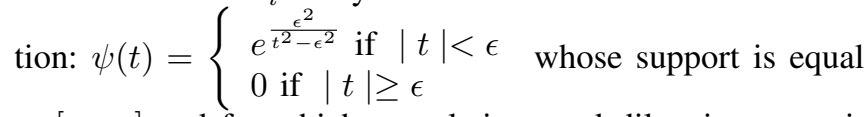
to $[-\epsilon, \epsilon]$ and for which, translations and dilatations permit to define test functions on any interval. In the numerical application, we took test functions whose supports are strictly included in the time interval $[0, T]$. This assumption implies, in particular, simplifications when we used integration by parts.

In that follows, an enclosure of $\int_{a}^{b} f(s) d s$ in the setmembership framework is obtained by the interval extension of the trapezoidal classical method and it is denoted $I_{[a, b]}(f)$ afterwards. The enclosures of the derivatives are obtained by using HOSM differentiators [4], [5]. The parameters of the HOSM differentiators are given by $\lambda_{0}=3, \lambda_{1}=0.2$ and $\lambda_{2}=0.1$.

\section{A. Numerical results}

For the simulation tests in Matlab, we choose constant parameter values given by $a_{1}=a_{2}=a_{3}=a_{4}=0.03$. The interval bounds for $\eta(t)$ are given by $[-0.10 .1]$.

The outputs are simulated on the time interval $[0,20]$ at the discrete times $\left(t_{i}\right)_{i=1, \ldots, M}$ such that the sampling period is equal to 0.1 . The faulty chosen scenario is given by the faults $f=(0.2 ; 0.05 ; 0.1 ; 0.1)$ occurring at $t=5 \mathrm{~s}$. For each fault, the initial intervals are given by $f_{0}=\left[\begin{array}{ll}0 & 5\end{array}\right]$ and the bisection threshold for the algorithm SIVIA is 0.025 .

For the two methods, the bounded-error fault estimation sets have an empty intersection with $[-\epsilon, \epsilon], \epsilon=0.025$ as soon as $t>5 s$.

For the first method, the fault estimation method was applied on the time interval $[5,20]$ and the computational time is 24.0036 seconds.

For the second one, the support length of the test functions being equal to 2.9 , the estimation procedure is done as soon as their supports are included in $[5 ; 20]$ which corresponds to $t>6.5 \mathrm{~s}$ and the computational time is 16.41 seconds.

In Tables 1 and 2, we give the percentage of eliminated initial fault box compared to the domains $\overline{\mathbb{S}}$ (solution and undetermined boxes) and $\underline{\mathbb{S}}$ (solution boxes only) respectively for the two methods. The eliminated percentage $\% \mathbf{f}$ is calculated by $\% \mathbf{f}=1-\frac{w([\mathbf{f}])}{w\left(\left[\mathbf{f}_{0}\right]\right)}$ where $w([\alpha])$ means the width of the interval $[\alpha]$.

In both Tables 1 and 2, the eliminated percentage of initial fault box is higher using the second method. Interestingly, the computational time is also smaller using the second method (divided by 1.5 ).

\section{Conclusion}

This paper proposes a fault detection and estimation method based on integro-differential polynomials obtained via the use of the distribution theory. This method provides

\begin{tabular}{ccc}
\hline Fault & $\% \mathbf{f}_{\text {method } 1}$ & $\% \mathbf{f}_{\text {method } 2}$ \\
\hline$f_{1}$ & 82.00 & 96.00 \\
\hline$f_{2}$ & 85.00 & 92.00 \\
\hline$f_{3}$ & 80.00 & 90.00 \\
\hline$f_{4}$ & 72.00 & 94.00 \\
\hline
\end{tabular}

TABLE I

ELIMINATED PERCENTAGE OF INITIAL FAULT BOX FOR THE TWO METHODS (UNDETERMINED AND SOLUTION BOXES).

\begin{tabular}{ccc}
\hline Fault & $\% \mathbf{f}_{\text {method } 1}$ & $\% \mathbf{f}_{\text {method } 2}$ \\
\hline$f_{1}$ & 92.10 & 96.20 \\
\hline$f_{2}$ & 90.10 & 95.20 \\
\hline$f_{3}$ & 91.60 & 93.10 \\
\hline$f_{4}$ & 87.20 & 93.90 \\
\hline
\end{tabular}

TABLE II

ELIMINATED PERCENTAGE OF INITIAL FAULT BOX FOR THE TWO METHODS (SOLUTION BOXES).

very good results in the case of the water-tank problem contrary to the classical input-output polynomials. The explanation for the improved results is that the integro-differential polynomials contain derivatives of smaller order. Furthermore, integration permits to annihilate part of the uncertainty. A first perspective of this work will be to applied this method on a more complex system for which the classical method fails. A second one is to better understand the role of test functions in this method and how can we choose them with respect to the noise.

\section{REFERENCES}

[1] F. Boulier, D. Lazard, F. Ollivier and M. Petitot, Computing representation for radicals of finitely generated differential ideals, Technical report, IT-306, Université Lille I, LIFL, 59655, Villeneuve d'Ascq, 1997.

[2] C. Jauberthie, N. Verdière and L. Travé-Massuyès, Fault detection and identification relying on set-membership identifiability, Annual Reviews in Control, 2013, Vol. 37, pp. 129-136.

[3] L. Jaulin, E. Walter, Set inversion via interval analysis for nonlinear bounded-error estimation, Automatica, 1993, Vol. 29, pp. 1053-1064.

[4] A. Levant, Higher-order sliding modes, differentiation and outputfeedback control, International Journal of Control, 2003, Vol. 76, pp. 924-941.

[5] L. Ravanbod, C. Jauberthie, N. Verdière, L. Travé-Massuyès, Improvements in enclosing uncertain derivatives and controlling ill-conditioned problems. Application to interval-based fault detection and isolation, Journal of Process Control, 2017, Vol. 58, pp. 139-151, 2017.

[6] H. Sira-Ramirez, C. Garcia Rodriguez, J. Cortes Romero and A. Luviano Juárez, Algebraic Identification and Estimation Methods, Feedback Control Systems (Book style), Wiley, 2014.

[7] M. Staroswiecki, G. Comtet-Varga, Analytical redundancy relations for fault detection and isolation in algebraic dynamic systems, Automatica, 2001, Vol. 37, pp. 687-699.

[8] N. Verdière, C. Jauberthie, L. Travé-Massuyès, Improvements in bounded error parameter estimation using distribution theory, Proceedings of ECC 2018, Kos, Cyprus, 12-15 juin, 2018.

[9] N. Verdière, C. Jauberthie, L. Travé-Massuyès, Functional diagnosability and detectability of nonlinear models based on analytical redundancy relations, Journal of Process Control, Septembre 2014, Volume 35, 1-10.

[10] Q. Zhang, M. Basseville, A. Benveniste, Fault detection and isolation in nonlinear dynamic systems: a combined input-output and local approach, Automatica, 1998, Vol. 34(11), pp. 1359-1373 